\title{
Plasmonic Bragg Reflector and Tamm Plasmon Polaritons in Metal-Dielectric Superlattices
}

\author{
S.M. VuKOVIC* \\ Institute of Physics, University of Belgrade, Pregrevica 118, 11080 Zemun, Belgrade, Serbia
}

\begin{abstract}
The properties of electromagnetic waves in two-dimensional periodic structures created by alternating metal and dielectric layers are investigated. The structure is assumed to be finite in the direction normal to the layers and infinite in other directions. Dispersion relations for both TE- and TM-polarized eigenmodes of metal-dielectric superlattice have been analyzed. It is demonstrated that the dispersion show typical properties of optic Tamm states.
\end{abstract}

PACS numbers: 73.20.Mf, 42.25.Bs, 42.70.Qs, 78.67.Pt

\section{Introduction}

Recently, it has been demonstrated both theoretically [1-3] and experimentally [4] that the surface states, termed optic Tamm states (or Tamm plasmon polaritons), can be formed in both TE- and TM-polarization at the interfaces inside photonic crystal heterostructures, as well as between a semi-infinite metal and a conventional dielectric Bragg reflector [3]. Unlike the conventional surface plasmon polaritons (SPPs), where the electromagnetic field is confined to the metal-dielectric interface via total internal reflection in the dielectric, fields of the optic Tamm states (OTSs) decay away from the interface due to the Bragg reflection. Also, in contrast to SPPs that are strictly TM-polarized with the dispersion that lies outside the light cone, OTSs can be both TE- and TM-polarized with the dispersion inside the light cone. Thus, OTSs can be excited directly by propagating waves which makes their significant advantage in many applications, as compared to SPPs.

In the present work, it is shown that eigenstates of a plasmonic Bragg mirror [5] that can be excited by almost normally incident light, possess the dispersion properties of OTSs. Their in-plane dispersion is parabolic, and the splitting between TE- and TM-polarized modes increases quadratically with the in-plane wave vector $[2,4]$. It is revealed that the group velocity of TE-polarized modes is always positive or zero, while the group velocity of TM-modes can be either positive, zero or negative. This leads to slow and spatially localized light with possible positive (both TE- and TM-polarized modes) or negative refraction (TM-polarization only).

\section{Formulation of the problem}

Let us consider a metal-dielectric superlattice core that consists of alternating $N$ metallic and $N-1$ dielectric layers embedded into dielectric cladding, as presented in Fig. 1. For the purpose of the present paper,

\footnotetext{
* corresponding author; e-mail: svukovic@phy.bg.ac.rs
}

all $N-1$ dielectric layers and the cladding are assumed to be the air, without loss of generality. Thus, a binary unit cell of this layer structure is formed by two layers: a metallic one with the thickness $a_{1}$ and permittivity $\varepsilon_{1}=1-\omega_{\mathrm{p}}^{2} / \omega^{2}<0$, and a dielectric one with the parameters $a_{2}$ and $\varepsilon_{2}=$ const $>0$, respectively. Here, $\omega_{\mathrm{p}}$ is the metal plasmon frequency, $\omega$ is the frequency of light, and the dielectric is supposed to be non-dispersive. The layered media has the period $L=a_{1}+a_{2}$. Assuming that the electric field of the $s$-polarized (TE) mode and the magnetic field of the $p$-polarized (TM) mode in the form: $\approx A_{q}(z) \exp (\mathrm{i} k x+\mathrm{i} q z-\mathrm{i} \omega t) \boldsymbol{e}_{y}$, the dispersion of the Bloch modes in the infinite layered media is described by the well known equation for bulk Bloch waves that comes from the eigenvalue problem for two layer $2 \times 2$ transfer matrix $T_{i j}[6]$ :

$$
\begin{aligned}
& \cos (q L)=(1 / 2)\left(T_{11}+T_{22}\right), \\
& \cos (q L)=\cos \left(\kappa_{1} \delta L\right) \cos \left(\kappa_{2}(1-\delta) L\right) \\
& -\frac{\left(1+\alpha_{s, p}^{2}\right)}{2 \alpha_{s, p}} \sin \left(\kappa_{1} \delta L\right) \sin \left(\kappa_{2}(1-\delta) L\right), \\
& 0<\delta=a_{1} / L<1,
\end{aligned}
$$

where $\kappa_{1,2}=\left(\frac{\omega^{2}}{\omega_{\mathrm{p}}^{2}} \varepsilon_{1,2}-k^{2}\right)^{1 / 2}, q$ is the Bloch wave number, and $\boldsymbol{k}$ the wave vector parallel to metal-dielectric interface. Notice that all spatial dimensions are normalized to $k_{\mathrm{p}}^{-1}$, and wave numbers to $k_{\mathrm{p}}=\omega_{\mathrm{p}} / c$. The polarization dependent coefficients are defined as $\alpha_{s}=\kappa_{1} / \kappa_{2}$ and $\alpha_{\mathrm{p}}=\kappa_{1} \varepsilon_{2} / \kappa_{2} \varepsilon_{1}$. There exist surface modes supported by each individual interface, or layer within the layered media, but the coupling (weak or strong) of these modes gives birth to new collective modes that propagate in the same direction, i.e. perpendicular to the Bloch wave vector. In the regime of strong coupling, when the unit cell thickness $L$ is much smaller than the free space wavelength $2 \pi \omega / c$, the optical properties of a multilayered structure can be described by the effective permittivity diagonal tensor that leads to the uniaxial crystal approximation [7]. However, here the problem will be solved exactly, without any assumption that regards $L, k$ 
and $q$. In particular, we will be interested in the dispersion domains where the group velocity of the mode(s) appears to be negative, which is a necessary and sufficient condition for negative refraction. Before proceeding to the results, it is necessary to mention that in a single metallic film surrounded by the air $[8,9]$ negative group velocity appears in the antisymmetric mode if the film is sufficiently thin: $L<1.46$. Both symmetric and antisymmetric modes are slow, i.e. their phase velocities are less than the velocity of light. On the other hand, for a single air gap between infinitely thick metallic claddings [9], negative group velocity is always present in the symmetric mode. This mode can be fast (for $k<\omega / \omega_{\mathrm{p}}$ ), with the phase velocity greater than the velocity of light. Unfortunately, strong attenuation takes place in that region, since $\varepsilon_{1} \simeq 0$. It is our aim to show that in the stratified metal-dielectric media it is possible to get fast modes for frequencies well below metal plasmon polariton frequency $\omega_{\mathrm{p}}$, and thus, low losses.

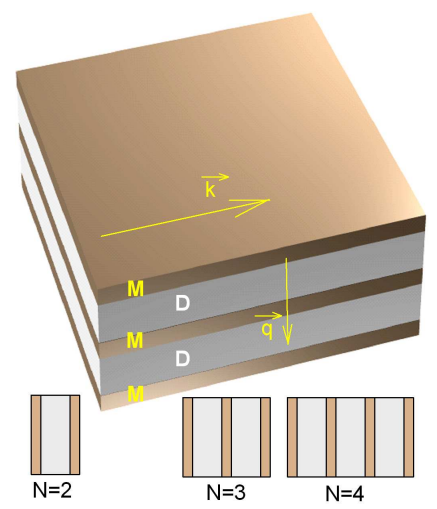

Fig. 1. Geometry of the multilayer structure; M stands for a metal layer, $\mathrm{D}$ for a dielectric one. $\mathrm{N}$ denotes the number of metal strata.

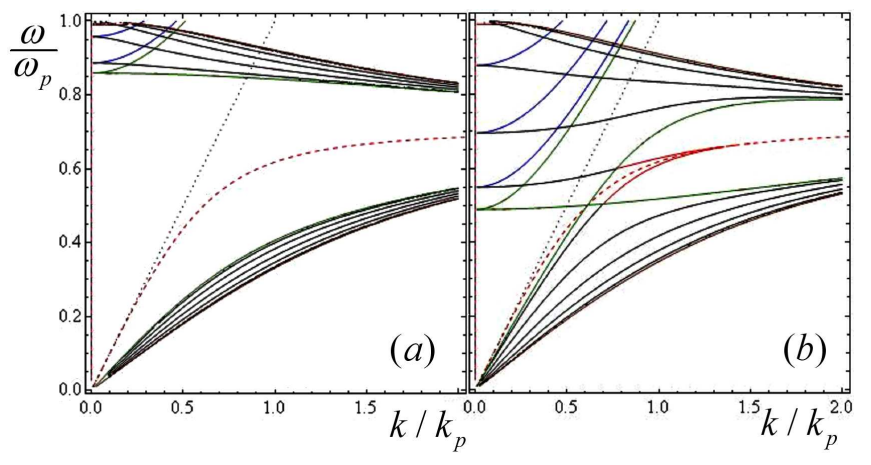

Fig. 2. Dispersion diagrams for $N=5$ plasmonic Bragg reflector and (a) $L=2, \delta=0.75$, (b) $L=2$, $\delta=0.25$. Black lines - TM modes; blue lines - TE modes; dashed - conventional surface plasmon polariton; red lines - TM modes in the "forbidden" zone; green lines - edges of the allowed bands: $\cos (q L)=1$; brown lines - edges of the allowed bands $\cos (q L)=-1$.

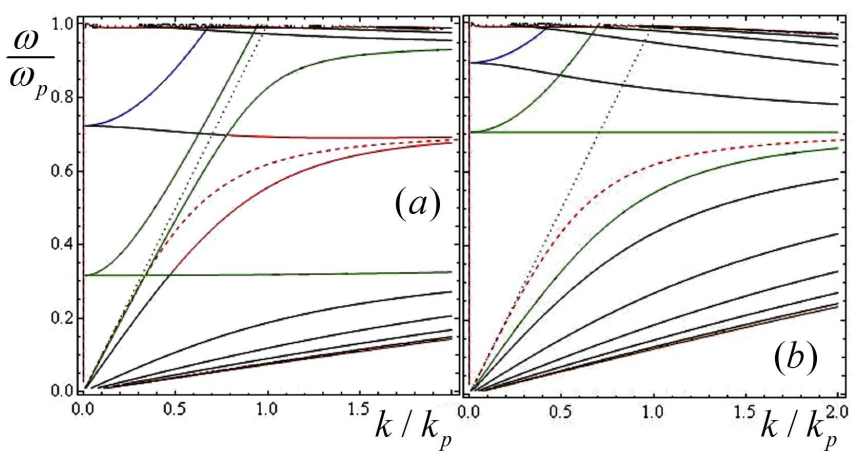

Fig. 3. Dispersion diagrams for $N=5$ plasmonic Bragg reflector and (a) $L=0.5, \delta=0.5$, (b) $L=0.5$, $\delta=0.1$. Everything else, as in Fig. 2 .

\section{Results}

The waveguide with $N$ metallic and $N-1$ dielectric layers has been studied in Ref. [10]. However, dispersion properties of the structure that is presented in Fig. 1, have not been investigated. By using the standard transfer matrix method [6], dispersion equation can be written in the following form:

$$
\frac{1}{2}\left(T_{11}-T_{22}\right) \frac{\sin (N q L)}{\sin (q L)}+\cos (N q L)=0 .
$$

This equation is now solved together with Eq. (1) and the corresponding dispersion diagrams (normalized frequency $\omega / \omega_{\mathrm{p}}$ versus normalized wave number $k$ ) are presented in Figs. 2 and 3. As can be seen, in the region of our interest $\left(\omega<\omega_{p}\right)$ there exist two allowed bands bounded by the curves $\cos (q L)= \pm 1$. There are exactly $N$ TM-polarized modes in each allowed band, and $N$ TE polarized modes in the upper allowed band only. TE and TM modes coalesce in the upper allowed band for $k=0$ that corresponds to the normal incidence of light on the multilayer structure. TE modes exist only within the light cone, while the TM modes in the lower allowed band exist only outside it. In the upper allowed band, however, TM modes cross the light cone line. The shape of dispersion curves strongly depends on the size of the unit cell $L$, as well as on the filling factor $\delta$. It comes out of Figs. 2b and 3b that for high $\delta$ there is no crossing into the gap, and all $2 N$ modes of the TM-polarization lie within allowed bands. This is in contrast to the case of low $\delta$ (see Figs. 2a and 3a), when the lowest mode of the upper band and the highest mode of the lower band cross into the Bragg gap and later, for higher values of $k$, coalesce into the single surface plasmon polariton. The group velocity of all modes in the lower band is always positive. In the upper band, first of all, group velocity tends to zero when $k \rightarrow 0$. In that case, the shape of all modes is parabolic and splitting between TE and TM modes increases quadratically with $k$. This confirms the existence of OTSs, as slow and spatially confined light in a plasmonic Bragg reflector configuration. Moreover, for sufficiently low $L$ and low $\delta$ the group velocity of the 
lowest mode in the upper allowed band becomes negative well bellow the plasma resonance, avoiding severe losses.

\section{Acknowledgments}

The author would like to acknowledge support by the Ministry of Science of the Republic of Serbia, under the project OI 141031.

\section{References}

[1] A.V. Kavokin, I.A. Shelykh, G. Malpuech, Phys. Rev. B 72, 233102 (2005).

[2] M. Kaliteevski, I. Iorsh, S. Brand, R.A. Abram, J.M. Chamberlain, A.V. Kavokin, I.A. Shelykh, Phys. Rev. B 76, 165415 (2007).

[3] X. Kang, W. Tan, Z. Wang, H. Chen, Phys. Rev. A 79, 043832 (2009).
[4] M.E. Sasin, R.P. Seisyan, M.A. Kalitteevski, S. Brand, R.A. Abram, J.M. Chamberlain, A.Yu. Egorov, A.P. Vasil'ev, V.S. Mikhrin, A.V. Kavokin, Appl. Phys. Lett. 92, 251112 (2008).

[5] J. Elser, V.A. Podolsky, Phys. Rev. Lett. 100, 066402 (2008).

[6] P. Yeh, A. Yariv, C.-S. Hong, J. Opt. Soc. Am. 67, 423 (1977).

[7] S.M. Rytov, Zh. Eksp. Teor. Fiz. 29, 605 (1955) [Sov. Phys. JETP 2, 466 (1956)].

[8] A.A. Oliner, T. Tamir, J. Appl. Phys. 33, 231 (1962).

[9] E.N. Economou, Phys. Rev. 182, 539 (1969).

[10] I. Avrutsky, I. Salakhutdinov, J. Elser, V. Podolskiy, Phys. Rev. B 75, 241402(R) (2007). 